\title{
CORRECTION OF A GENERALIZATION OF A THEOREM OF BEURLING AND LIVINGSTON
}

\author{
PETER A. FOWLER
}

\begin{abstract}
A generalization of a Riesz-Fischer theorem proved by Beurling and Livingston for smooth uniformly convex Banach spaces also holds for smooth, strictly convex, reflexive Banach spaces. THEOrEm. Let $B$ be $a$ smooth, strictly convex, reflexive Banach space. Let $T: B \rightarrow B^{*}$ be a duality map, $C$ a closed subspace of $B, h \in B, k \in B^{*}$. Then $T(C+h) \cap\left(C^{\perp}+k\right)$ is a single point. A two-dimensional counterexample shows that $T(C+h) \cap$ $\left(C^{\perp}+k\right)=\varnothing$ is possible if $B$ is not smooth, contrary to the claim of Theorem 4 of Browder, On a theorem of Beurling and Livingston, Canad. J. Math. 17 (1965), 367-372.
\end{abstract}

Introduction. Beurling and Livingston [1, Theorem 2], presented a generalization of the Riesz-Fischer theorem using single-valued duality map from a uniformly convex, smooth Banach space $B$ to its conjugate space $B^{*}$. The paper [2, Theorem 4] attempted to extend the result to single-valued duality maps on strictly convex reflexive Banach spaces without the hypothesis of smoothness. This extended result was cited in the paper [3, p. 338], which dealt primarily with multi-valued duality maps. The present paper demonstrates with a counterexample that the claim of [2] is in error, but that if the hypothesis of smoothness is restored, then the theorem can be proved along the lines of [2].

1. Preliminaries. We follow the definition and notation of $[2, \S 2]$. Denote by $B$ a Banach space and let $S=\{u \in B \mid\|u\|=1\}$ be the unit sphere.

Definition. A duality map is a single-valued map $T: B \rightarrow B^{*}$ which verifies

$$
\langle u, T(u)\rangle=\|u\| \cdot\|T(u)\| \quad \text { for all } u \in B,
$$

and for some continuous, strictly increasing $\phi: R^{+} \rightarrow R^{+}$with $\phi(0)=0$ and $\lim _{r \rightarrow \infty} \phi(r)=\infty$,

$$
\|T(u)\|=\phi(\|u\|) \text { for all } u \in B \text {. }
$$

Recall that a Banach space is smooth at $u \neq 0$ if $S$ has a unique supporting hyperplane at $u /\|u\|$, or equivalently, the Gateaux differential

$$
G(w, u)=\lim _{t \rightarrow 0} \frac{\|u+t w\|-\|u\|}{t}
$$

exists for all $w \in B$. See for example James [4, p. 239]. It is immediate that for a given $\phi$, a Banach space is smooth at $u \neq 0$ iff the value at $u$ of any

Received by the editors June 10, 1975 and, in revised form, January 16, 1976 and February 24, 1978.

AMS (MOS) subject classifications (1970). Primary 46B10. 
duality map corresponding to $\phi$ is uniquely determined by the formula

$$
\langle w, T(u)\rangle=\phi(\|u\|) \cdot G(w, u) \text { for all } w \in B .
$$

Thus, a Banach space is smooth (at all $u \neq 0$ ) iff the duality map corresponding to a given $\phi$ is unique.

2. Counterexample. It follows easily from the above discussion that part (b) of Lemma 2.3 in [2] is false. Also, part (c) of Lemma 2.3 and Theorem 4 in [2] are false, as is shown below.

Let $B=R^{2}, h=(1,0) \in R^{2}$. Let $\|\cdot\|$ denote any norm for which $\pm h \in$ $S$ and $S$ is not smooth at $\pm h$. Further, require that $\|\cdot\|$ be chosen so $\pm h$ are the only points of $S$ at which supporting lines for $S$ are parallel to the $y$-axis. (For example, the strictly convex norm

$$
\|(x, y)\|=|y|+\sqrt{x^{2}+y^{2}}
$$

gives a unit sphere $S=\left\{(x, y) \mid y= \pm\left(\frac{1}{2}-\frac{1}{2} x^{2}\right),-1 \leqslant x \leqslant 1\right\}$ with these properties.) For $\phi(r)=r$, let $T$ be any corresponding duality map for which $L=\left\{u \in R^{2} \mid\langle u, T(h)\rangle=1\right\}$ is a supporting line for $S$ at $h$ other than the line $x=1$. Let $C$ be the $y$-axis and $k$ the zero of $B^{*}$. Then $C+h$ is the line $x=1$.

We assert that

$$
T(C+h) \cap\left(C^{\perp}+k\right)=T(C+h) \cap C^{\perp}=\varnothing,
$$

which shows Theorem 4 in [2] to be false. In fact, $T(h) \notin C^{\perp}$ because $T(h) \in C^{\perp}$ implies $\langle c+h, T(h)\rangle=\langle h, T(h)\rangle=1$ for any $c \in C$. This is impossible since $T$ was chosen so that $C+h \neq L$. If $T(c+h) \in C^{\perp}$ for any nonzero $c \in C$, then

$$
\begin{aligned}
\|c+h\|^{2} & =\langle c+h, T(c+h)\rangle=\langle h, T(c+h)\rangle \\
& \leqslant\|h\| \cdot\|T(c+h)\|=\|c+h\|
\end{aligned}
$$

which entails $\|c+h\| \leqslant 1$. But $\|c+h\|>1$ must hold because $\pm h$ are the only points of $S$ at which supporting lines are parallel to $C$. Thus $T(c+h) \notin$ $C^{\perp}$, and the assertion is proved.

Further, it is clear that none of the infinitely many duality maps corresponding to $\phi(r)=r$ is continuous as a map into $B^{*}$ with the weak topology. Thus, Lemma 2.3(c) of [2] is false.

3. Corrections. The origin of the error in [2, Theorem 4] lies in the errors in [2, Lemma 2.3(b), (c)]. Corrected statements of (b) and (c) and of this theorem follow.

(b) A Banach space $B$ is smooth iff there is exactly one duality map $T$ corresponding to each function $\phi$ of the Definition.

(c) If $B$ is reflexive and smooth then the duality map $T$ corresponding to each $\phi$ is a continuous mapping of $B$ into $B^{*}$ with the weak topology. 
TheOREM (BEURLING-LivingSTON). Let $B$ be a reflexive, strictly convex and smooth Banach space, $T$ a duality map of $B$ into $B^{*}$. Let $C$ be a closed subspace of $B, h$ a fixed element of $B, k$ a fixed element of $B^{*}$. Then the set

$$
T(C+h) \cap\left(C^{\perp}+k\right)
$$

contains exactly one element.

That (b) is true follows from the discussion in $\$ 1$. The proof of (c) follows from (b) in a manner analogous to the argument in [2, p. 371]. The proof of the Theorem is then analogous to the argument in [2, p. 372].

\section{REFERENCES}

1. A. Beurling and A. E. Livingston, $A$ theorem on duality mappings in Banach spaces, Ark. Mat. 4 (1961), 405-411.

2. F. E. Browder, On a theorem of Beurling and Livingston, Canad. J. Math. 17 (1965), 367-372.

3. Multivalued monotone nonlinear mappings and duality mappings in Banach space, Trans. Amer. Math. Soc. 118 (1965), 338-351.

4. R. C. James, Linear functionals as differentials of a norm, Math. Mag. 24 (1951), 237-244.

Department of Mathematics, California State University, Hayward, California 94542 